\title{
Expectativas, consumo de alcohol
}

\section{y problemas asociados en estudiantes \\ universitarios de la ciudad de México}

\author{
Jazmín Mora-Ríos, M. en Psic., ${ }^{(1)}$ G uillermina N atera, M. en Psic. ${ }^{(2)}$
}

\section{Mora Ríos J, Natera G. Expectativas, consumo de alcohol y problemas asociados en estudiantes universitarios de la ciudad de México. Salud Publica Mex 2001;43:89-96.}

EI texto completo en inglés de este artículo está disponible en: http://www.insp.mx/salud/index.html

\section{Resumen}

Objetivo. Establecer la relación entre las expectativas hacia el consumo de alcohol, los patrones de su uso y los problemas asociados a su consumo en una muestra de estudiantes universitarios de la ciudad de México. Material y métodos. Estudio transversal realizado en octubre de 1998, en el que participaron 678 estudiantes, hombres y mujeres entre los 17 y 25 años de edad, provenientes de universidades públicas y privadas. En él se midieron las expectativas a partir de un cuestionario de autorreporte, el Alcohol Expectancy $Q$ uestionnaire (AEQ ), que fue adaptado a esta población obteniendo una consistencia interna glo bal elevada (alpha $=0.93$ ). Resultados. D el total de los estudiantes, $31 \%$ presentó un consumo alto (mayor a cinco copas por ocasión de consumo en el último año), principalmente en los varones, mientras que $17 \%$ fueron no consumidores. Mediante un modelo estructural de ecuaciones se estudió la relación entre las subescalas de expectativas, el patrón de consumo y problemas asociados. El análisis de varianza mostró una relación estadísticamente significativa entre seis subescalas de expectativas (el alcohol como facilitador de la interacción grupal, expresividad verbal, desinhibición, incremento de la sexualidad, reducción de la tensión psicológica e incremento de la agresividad) y las variables sexo, consumo de alcohol y problemas asociados $(F=5.23, g=1, p<0.05)$.

\author{
Mora Ríos J, Natera G. \\ Alcohol use expectancy, intake, \\ and related problems among college \\ students in Mexico City. \\ Salud Publica Mex 2001;43:89-96. \\ The English version of this paper \\ is available at: http://www.insp.mx/salud/index.html
}

\begin{abstract}
A bstract
Objective. To assess the relationship between alcohol use expectancies, drinking patterns, and alcohol-related problems, among college students in Mexico City. Material and methods. This cross-sectional study was conducted in 0 ctober 1998. Study subjects were 678 male and female college students aged between 17 and 25 years, from private and public schools. Alcohol expectancies were measured through the self-reported "Alcohol Expectancy Q uestionnaire" (AEQ), adapted for this population, with a high overall internal reliability coefficient (alpha $=0.93$ ). Results. Thirty-one per cent of study subjects, mainly males, reported heavy drinking ( 5 drinks or more per drinking occasion in the previous year), while $17 \%$ were non-consumers. Relationships for each of the AEQ scales, drinking patterns, and alcohol-related problems, were analyzed through a structural equations model. A nalysis of variance showed a statistically significant association between six AEQ subscales -alcohol as a facilitator of a group interaction, verbal expression, disinhibition, sexual enhancement, reduction of psychological tension and arousal agression$(F=5.23, d f=1, p<0.05)$. Conclusions. Study findings from other countries show that effects attributed to alcohol, in anticipation of the drinking episode (expectancies), are closely related to alcohol intake. 0 ur results showed that heavy
\end{abstract}

Una versión preeliminar de este trabajo se presentó en el XVI Congreso N acional de la A sociación Psiquiátrica Mexicana. Huatulco, 0 axaca, noviembre de 1999.

(1) Dirección de Investigaciones Epidemiológicas y Sociales, Instituto N acional de Psiquiatría Ramón de la Fuente Muñiz (IN P), México.

(2) Departamento de Investigaciones Psicosociales, IN P, México.

Fecha de recibido: 12 de junio de 2000 - Fecha de aprobado: 4 de diciembre de 2000

Solicitud de sobretiros: Psic. Jazmín Mora Ríos. Dirección de Investigaciones Epidemiológicas y Sociales, Instituto N acional de Psiquiatría. Calzada México-X ochimilco 101, colonia San Lorenzo Huipulco, Delegación Tlalpan, 14370 México, D.F., México.

Correo electrónico: morarj@ imp.edu.mx 
Conclusiones. De manera consistente con lo informado en otros países, se encontró que los efectos que las personas atribuyen al consumo de alcohol, anticipándose a la experiencia de estar bebiendo (expectativas), están estrechamente relacionadas con su consumo. Los resultados de este estudio indican que los estudiantes con un consumo alto de alcohol tuvieron mayores puntuaciones en seis de las subescalas de expectativas, además de que éstas fueron más elevadas entre quienes informaron que tuvieron problemas relacionados con el consumo en el último año. Las expectativas del alcohol como facilitador de la interacción grupal, como reductor de la tensión psicológica y como agente que incrementa la conducta agresiva se relacionaron con un consumo de alcohol más alto. El texto completo en inglés de este artículo está disponible en: http:// www.insp. $\mathrm{mx} / \mathrm{salud} /$ index.html

Palabras clave: cuestionario de expectativas hacia el alcohol (AEQ); consumo de bebidas alcohólicas; estudiantes; México drinkers had higher scores in six AEQ subscales, particularly those who reported having alcohol-related problems in the previous year. Alcohol expectancies related to higher alcohol intake were: facilitation of social interaction, decreasing psycholo gical tension, and increasing arousal/aggression. The English version of this paper is available at: http:// www.insp.mx/salud/index.html

Key words: alcohol expectancy questionnaire (AEQ); alcohol drinking; students; M exico
$\mathrm{U}$ na de las principales aportaciones de las aproximaciones psicosociales contemporáneas en el campo de las adicciones ha sido el estudio de las expectativas. Particularmente en el caso del alcohol, y con base en una perspectiva cognitivo-conductual, los esfuerzos teóricos y empíricos se han enfocado en la identificación temprana de los predictores del uso y abuso de alcohol.

Estudios de laboratorio han mostrado que las expectativas hacia el alcohol pueden afectar la cantidad de consumo. ${ }^{1,2}$ Por otra parte, las expectativas han sido estudiadas mediante instrumentos de autorreporte en investigaciones con población de estudiantes, principalmente adolescentes y adultos. ${ }^{3-10}$

Las expectativas se definen como "la anticipación de una relación sistemática entre eventos $\mathrm{u}$ objetos en una situación futura", es decir, si ciertos eventos son registrados, en consecuencia, ciertos eventos son esperados. Las expectativas pueden ser inferidas por tener un estatus causal en las que las propias acciones de un individuo producen una cierta consecuencia. ${ }^{2}$ En términos prácticos, las expectativas se refieren a las creencias individuales sobre los efectos esperados del consumo de alcohol y son un constructo teórico importante ya que permite vincular las experiencias tempranas con el alcohol y las decisiones que se tienen a futuro sobre el consumo de esta sustancia y, mediante su estudio, es posible identificar los factores sociales y cognitivos que se relacionan con un consumo problemático, lo que es fundamental para el desarrollo de una teoría comprehensiva del abuso de alcohol.

Las expectativas difieren en términos del género, la raza y la cultura de los respondientes. ${ }^{2,11}$ Se consi- dera que el desarrollo de las expectativas comienza con una serie de creencias globales, difusas e indiferenciadas sobre los efectos del alcohol, que tienden a incrementarse con la edad, la experiencia con el alcohol y la exposición familiar, social y cultural de las creencias acerca del alcohol, las cuales constituyen las fuentes primarias de aprendizaje de esta sustancia. ${ }^{12,13}$

Las expectativas intervienen en el inicio y mantenimiento de dicho consumo durante la adolescencia ${ }^{14}$ y se correlacionan con los patrones diferenciales de consumo no sólo en este periodo sino en adultos con diferentes características poblacionales. ${ }^{15,16}$ Es común que exista un incremento en el consumo de los estudiantes al ingresar a la universidad, por lo que es importante investigar, en este periodo, el papel que juegan estas expectativas en la transición a los patrones de consumo en la vida adulta.

En estudiantes universitarios se ha encontrado que las expectativas son mejores predictores concurrentes del comportamiento de consumo que las variables demográficas, que son conocidas por tener un poder predictivo sustancial. ${ }^{4}$ Las expectativas pueden variar de acuerdo con los hábitos de consumo; por ejemplo, se ha mostrado que los estudiantes cuyo consumo de alcohol es moderado tienen expectativas más relacionadas con las experiencias positivas respecto a los efectos del consumo y el realce de los placeres sociales, en tanto que los bebedores excesivos esperan que el alcohol incremente sus conductas sexual y agresiva, además de reducir la tensión a partir del consumo. ${ }^{3}$

En población universitaria se ha puesto de manifiesto que los estudiantes esperan que otras personas sean más fuertemente afectados por el alcohol 
de lo que esperan para sí mismos, tanto en aspectos positivos como negativos. ${ }^{7}$ La distinción entre efectos sociales e individuales permite predecir diferentes resultados de consumo; por ejemplo, las expectativas sociales predicen un consumo moderado mientras que las expectativas individuales predicen un consumo problemático. $^{8}$

En México el consumo de alcohol en estudiantes ha generado interés desde una óptica de salud pública por los problemas relacionados con su consumo. En población universitaria, los resultados de las encuestas revelan que la proporción de bebedores de alcohol y los problemas asociados aumentan con la edad. En 1980, el consumo alguna vez en la vida en esta población fue de $86.4 \%$ y los problemas más frecuentemente reportados fueron: el deseo de beber menos $(16 \%)$, los arrestos $(9.8 \%)$ y los problemas familiares $(5.6 \%) .{ }^{17}$

De acuerdo con los datos más recientes reportados por la Encuesta Nacional de Adicciones (1998), particularmente en la población entre los 18 a 29 años de edad, que habita en la ciudad de México, $78.6 \%$ de los hombres encuestados es bebedor actual, mientras que en las mujeres la proporción corresponde a 53.8\%. Las bebidas de mayor preferencia fueron la cerveza, los destilados y las bebidas embotelladas preparadas como los coolers. ${ }^{*}, 18$

Las actitudes han sido una de las variables psicosociales que más se han utilizado en la investigación sobre adicciones en población escolar; no obstante, se ha encontrado que las actitudes tienen escaso valor predictivo en el consumo de alcohol, siendo más determinantes las variables como sexo y cantidad de problemas asociados. Aun cuando las expectativas se relacionan con las actitudes, pero a diferencia de éstas, son creencias que tienen un componente de causa-efecto (por ejemplo, algunas personas consideran que beber alcohol les ayuda a tener valor para enfrentar situaciones difíciles). Las evidencias que sugieren su utilidad en la explicación del uso y abuso de alcohol están ampliamente documentadas. ${ }^{10,11,13,14}$ El estudio de las expectativas, conjuntamente con otras variables, como sexo, peso, talla, edad del consumidor, ocasiones y circunstancias de consumo, puede contribuir a un mayor conocimiento en la problemática de adicciones. ${ }^{18}$

Con base en lo anterior, en un estudio previo, ${ }^{19}$ se llevó a cabo la adaptación del cuestionario de expec-

\footnotetext{
* Secretaría de Salud. Encuesta Nacional de Adicciones: Alcohol. Población 18-65 años, México D.F.: SSA- CONADIC-IMP (2000). Documento interno.
}

tativas hacia el alcohol (AEQ) en población universitaria con el objeto de identificar las diferentes áreas que lo conforman. El propósito del presente trabajo es establecer la relación entre las subescalas identificadas de expectativas y el patrón diferencial de consumo de bebidas alcohólicas reportado en el último año en un grupo de estudiantes de universidades públicas y privadas de la ciudad de México. Asimismo, se incluyeron los problemas asociados al alcohol como un indicador de consumo problemático que podría estar relacionado con las expectativas.

\section{Material y métodos}

Se realizó un estudio transversal exposfacto; en el cual se obtuvo un muestreo diversificado por cuotas en universidades públicas $(52.2 \%)$ y privadas $(47.5 \%)$ de la ciudad de México, considerando diferentes áreas de estudio, como ciencias biológicas $(20 \%)$, artísticas $(19 \%)$, ciencias económico-administrativas $(12 \%)$, ciencias exactas $(14.5 \%)$ y ciencias sociales $(34 \%)$. Asimismo, se estableció un control por sexo y tipo de universidad.

El tamaño de la muestra se calculó con base en el número de reactivos contenidos en la versión original del $\mathrm{AEQ}^{19}$ que se requieren para obtener la validez factorial y confiabilidad del instrumento; se calcularon cinco aplicaciones por reactivo incluyendo un margen para considerar a los casos omitidos.

Participaron 678 estudiantes, de sexo masculino y femenino, cuyas edades oscilaban entre los 17 y 25 años, la edad promedio fue 20 años, $(\mathrm{DE}=1.80)$ (cuadro I).

Respecto a los instrumentos utilizados, se aplicó un cuestionario que contenía la siguiente información:

Datos sociodemográficos. Se incluyen variables como sexo, edad, ocupación, estado civil, semestre actual de estudios, lugar de residencia y tipo de escuela (pública-privada).

Cuestionario de expectativas hacia el alcohol (AEQ). ${ }^{3}$ Mide las creencias positivas del consumo de alcohol y sus efectos en la conducta social y emocional alrededor de seis dimensiones: a) transforma positivamente las experiencias; b) incrementa sexualidad; c) realza el placer físico y social; d)aumenta la asertividad social; e) reduce la tensión, y f) incrementa el poder y la agresión. Estas dimensiones indagan los efectos positivos que se espera obtener bajo el efecto de cantidades moderadas de alcohol, por ejemplo: "unas cuantas copas" o "un par de copas" y es respondido con base en un formato dicotómico (cierto-falso) en función de las 


\section{Cuadro I \\ Características sociodemográficas \\ DE LOS PARTICIPANTES EN EL ESTUDIO \\ SOBRE EXPECTATIVA Y CONSUMO DE ALCOHOL. Instituto Nacional de Psiquiatría, México, 1998}
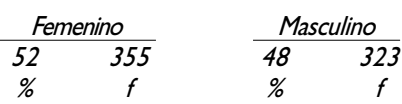

Edad

\begin{tabular}{rrrrr}
$17-19$ años & 38 & 133 & 25 & 81 \\
\hline $20-22$ años & 54 & 193 & 52 & 168 \\
\hline $23-25$ años & 8 & 29 & 23 & 74
\end{tabular}

Universidad

\begin{tabular}{lllll} 
Pública & 53 & 187 & 51 & 166 \\
\hline Privada & 47 & 168 & 49 & 157
\end{tabular}

O cupación

\begin{tabular}{lrrrr} 
Estudia & 83 & 294 & 73 & 237 \\
\hline Estudia y trabaja & 17 & 61 & 27 & 86
\end{tabular}

Semestre actual

\begin{tabular}{lrrrr}
$1-2$ & 31 & 109 & 39 & 125 \\
\hline $3-4$ & 29 & 103 & 29 & 93 \\
\hline $5-6$ & 34 & 121 & 21 & 68 \\
\hline 0 tros (tesista) & 6 & 22 & 11 & 37
\end{tabular}

creencias personales, sentimientos y experiencias del respondiente en relación con el alcohol.

Se utilizó la versión para adultos del AEQ y fue adaptada en población universitaria de la ciudad de México. Se identificaron ocho subescalas de las expectativas: a) el alcohol facilita la interacción grupal; b) favorece la expresividad verbal; c) desinhibe la conducta; d) incrementa la sexualidad; e) reduce la tensión física; f) reduce la tensión psicológica, g) incrementa la agresión y los sentimientos de poder, y h) propicia los cambios psicofisiológicos. La escala obtuvo una consistencia interna global muy elevada (alpha de Cronbach $=0.93) .{ }^{20}$

Hábitos de consumo de alcohol. Se utilizaron los criterios propuestos por la Encuesta Nacional de Adicciones ${ }^{20}$ para medir la frecuencia de consumo de diferentes tipos de bebidas (cerveza, vino de mesa, destilados y bebidas preparadas, como coolers y viña real). Se obtuvo la frecuencia de consumo de las diferentes cantidades de alcohol por ocasión de consumo en el último mes y año. Para efectos de este estudio se obtuvo el patrón de consumo en el último año y se dividió en las siguientes categorías: a) no consumidores, b) consumidores de menos de cinco copas (consumo bajo), y c) consumidores de cinco copas o más por ocasión (consumo alto).

Asimismo, se incluyó información de otras variables relacionadas como lugares de consumo y problemas asociados con el consumo (físico, accidentes de tránsito, personales, con la policía).

Procedimiento. La aplicación del instrumento tuvo lugar en las aulas de clase, en el transcurso de 45 minutos, previo consentimiento de las autoridades. Participaron cuatro entrevistadoras, pasantes de las licenciaturas de Psicología y Sociología, previamente capacitadas en el manejo de los instrumentos, quienes solicitaron la colaboración voluntaria de los estudiantes para que trataran de responder lo más sincera y espontáneamente posible acerca de sus creencias, sentimientos y opiniones personales hacia el alcohol. Los estudiantes respondieron los cuestionarios individualmente en los salones de clase. Se garantizó el anonimato y la confidencialidad de la información.

Para el análisis de la información se utilizó un modelo estructural de ecuaciones ${ }^{21}$ y un análisis de varianza (Anova) contenido en el paquete estadístico SPSS (versión 6.1.2) para windows.

\section{Resultados}

Consumo de alcohol y otras variables relacionadas. Los varones iniciaron su consumo de alcohol en promedio a los 14 años $(\mathrm{DE}=2.6)$, mientras que en las mujeres el inicio fue a los 15 años en promedio ( $\mathrm{DE}=2.9)$. Las principales bebidas consumidas por los estudiantes bajo la categoría de "alguna vez en la vida" fueron los cocteles y las bebidas como viña real ( $86 \%)$, lo que es de esperarse puesto que son bebidas dirigidas a los jóvenes y son principalmente consumidas por las mujeres; es importante enfatizar que este tipo de bebidas, que comúnmente son consideradas como refrescantes, también pueden producir adicción. Los destilados (85\%) y la cerveza (83\%) fueron mencionados en segundo y tercer lugar, respectivamente.

Llama la atención el uso del pulque, 31\% de los jóvenes reportaron su consumo, lo cual es elevado si se consideran los resultados de estudios previos que indican que el pulque no es el tipo de bebida que se utiliza comúnmente en población juvenil urbana.

Los estudiantes de sexo masculino consumen en mayor frecuencia y cantidad que las mujeres. Alrededor de $30 \%$ de las mujeres bebe en promedio de una a cuatro copas por ocasión con una frecuencia de al menos una vez al año, mientras que $22.2 \%$ de los hom- 
bres consume entre cinco y 11 copas al menos una vez a la semana. No obstante, no hay que menospreciar que $7 \%$ de las jóvenes presentan un consumo mayor a cinco copas y que lo hacen al menos una vez a la semana y una vez al mes.

Con base en los patrones diferenciales de consumo de alcohol informados en el último año en la población estudiada se encontró que poco más de la mitad de los estudiantes (52\%) presenta un consumo moderado de alcohol, menor a cinco copas por ocasión de consumo. El 31\% de los jóvenes, principalmente varones, presenta un consumo de cinco copas o más por ocasión. La proporción fue de tres hombres por una mujer con consumo alto. Tan sólo 17\% de los estudiantes no consumen ningún tipo de bebida alcohólica (figura 1).

En el cuadro II se muestran los patrones de uso de alcohol por sexo y edad. En las mujeres se observa una mayor tendencia hacia un patrón de consumo de alcohol moderado. Sin embargo, al considerar el consumo alto $6 \%$ de éstas se ubica entre los 20 y 22 años de edad, mientras que en los hombres corresponde al rango de 23 a 25 años (14\%).

Respecto al patrón de consumo por tipo de escuela, se observó un consumo ligeramente más elevado en mujeres de universidades privadas (17.9\%) que en las jóvenes de universidades públicas (11.8\%). Por otra parte, el consumo más alto en los varones corresponde a los estudiantes de universidades públicas en comparación con las privadas, aunque estas diferencias no fueron estadísticamente significativas.

En cuanto a los lugares donde beben los jóvenes, éstos ingieren alcohol principalmente en restaurantes $(80.9 \%)$, en reuniones familiares $(74.6 \%)$ y en fiestas escolares (52\%). El lugar menos habitual es en eventos deportivos.

De los jóvenes, $26 \%$ mencionó tener problemas asociados al consumo, principalmente los hombres $(17.9 \%)$ en comparación con $8.2 \%$ de las mujeres reportaron estos problemas; los más importantes fueron con las relaciones interpersonales, con la policía y con la salud física, seguidos por los accidentes que, como era de esperarse, en todos los casos los hombres obtuvieron puntuaciones más altas que las mujeres. En el cuadro III se muestra la relación entre el patrón de consumo y los problemas asociados, hay una tendencia a informar mayores problemas asociados al alcohol en los jóvenes que presentan un consumo más elevado.

\section{Expectativas y consumo de alcohol}

Se utilizó un modelo estructural de ecuaciones ${ }^{21}$ con el objeto de estudiar la relación entre las subescalas de expectativas, el patrón de consumo y los problemas asociados con la ingesta de alcohol en el último año.

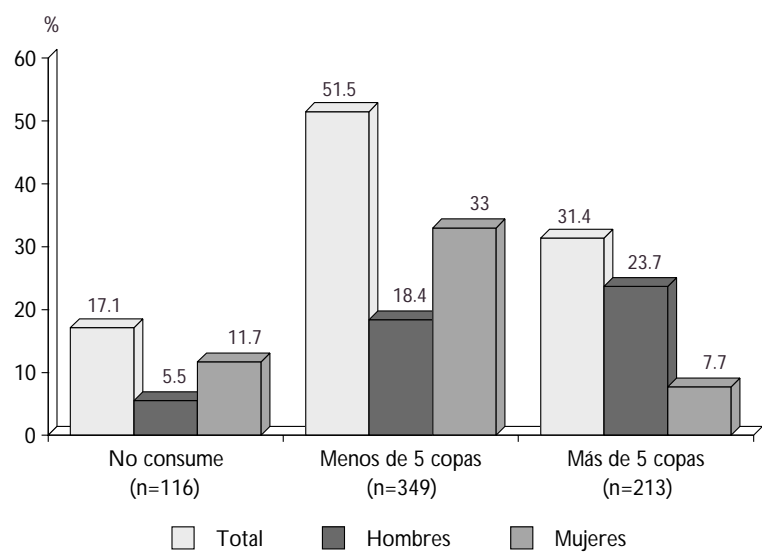

Figura 1. Consumo de alcohol en estudiantes UNIversitarios, según sexo. Instituto Nacional de PsiQUiatría, MéXICO, 1998

\section{Cuadro II \\ Consumo de alcohol en el último año SEGÚN SEXO Y GRUPOS DE EDAD. Instituto Nacional de Psiquiatría, México, 1998}

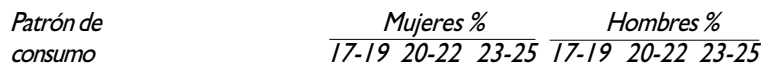

\begin{tabular}{lrrrrrr} 
Número de consumidores & 8 & 25 & 4 & 5 & 5 & 10 \\
\hline Menos de 5 copas & 13 & 32 & 10 & 10 & 21 & 26 \\
\hline Más de 5 copas & 1 & 6 & 1 & 10 & 8 & 14
\end{tabular}

$n=678$

Para llevar a cabo este procedimiento se eligió únicamente a la población de consumidores (alto-moderado) y problemas asociados, contrastando a quienes informaron haber tenido estos problemas en el último año y a quienes no los tuvieron. El mejor ajuste del modelo se obtuvo al eliminar a las subescalas de expectativas que obtuvieron cargas factoriales muy bajas; en este caso fueron de 0.29 para la subescala de "cambios psicofisiológicos" y de 0.41 que correspondió a la "tensión física", obteniendo una $X^{2}{ }_{S B}=86.44$, $(\mathrm{gl}=66), p=0.000$, RCFI (Robust Comparative Fit In$\operatorname{dex})=0.97$, RMSEA (Root Means SQ Error of App)= 0.050. Asimismo, se encontró que las expectativas sobre "agresividad y sentimientos de poder" están muy relacionadas con las expectativas grupales, mientras que las expectativas sobre desinhibición están relacionadas con las sexuales.

Por último, se llevó a cabo un análisis de varianza con seis de las subescalas de las expectativas que se relacionaron con el consumo (moderado vs alto) y con 


\begin{tabular}{|c|c|c|c|c|c|c|}
\hline $\begin{array}{l}\text { Patrón de consl } \\
\text { Instituto Nacional }\end{array}$ & $\begin{array}{l}\text { Cuac } \\
\text { MO Y } \\
\text { DE I }\end{array}$ & $\begin{array}{l}\text { III } \\
\text { OOBL } \\
\text { QUIA }\end{array}$ & $\mathbf{R} \mid \mathbf{A}$, & MÉX & $\begin{array}{l}\text { ADO } \\
\text { O, }\end{array}$ & \\
\hline Tipo de & const & dores & $\begin{array}{r}M \\
\text { de } 5 \\
\end{array}$ & $\begin{array}{l}\text { los } \\
\text { opas }\end{array}$ & $\begin{array}{r}N \\
\text { de } 5 \\
\end{array}$ & opas \\
\hline problema & $f$ & $\%$ & $f$ & $\%$ & $f$ & $\%$ \\
\hline Relaciones personales*** & 8 & 1.2 & 47 & 6.9 & 83 & 12.2 \\
\hline Problemas con la policía*** & 4 & 0.6 & 20 & 2.9 & 40 & 5.9 \\
\hline Salud física*** & 5 & 0.7 & 23 & 3.4 & 35 & 16.4 \\
\hline $\begin{array}{l}\text { Accidentes*** } \\
\begin{array}{l}* \\
\text { p }-0.000 \\
\text { p }-0.005\end{array}\end{array}$ & 2 & 0.3 & 15 & 2.2 & 29 & 4.3 \\
\hline
\end{tabular}

los problemas asociados. La variable sexo también fue considerada en este análisis (cuadro IV).

Se encontró una relación significativa entre las subescalas de expectativas del AEQ "expresividad verbal" $(\mathrm{F}=22.72, \mathrm{gl}=1, p<0.001)$, "desinhibición" ( $\mathrm{F}=15.97, \mathrm{gl}=1, p<0.001)$, "incremento de la sexualidad" $(\mathrm{F}=41.42, \mathrm{gl}=1, p<0.001)$, "interacción grupal” ( $\mathrm{F}=69.25$, $\mathrm{gl}=1, p<0.001)$, "reducción de la tensión psicológica" ( $\mathrm{F}=68.82, \mathrm{gl}=1, p<0.001)$, "incremento de la agresividad y sentimientos de poder" ( $\mathrm{F}=31.63, \mathrm{gl}=1, p<0.001)$ y el patrón de consumo. Los consumidores altos obtuvieron las puntuaciones más elevadas en las subescalas de expectativas que los moderados. En los estudiantes que presentaron consumo alto, las principales expectativas fueron el alcohol como facilitador de la interacción grupal, como reductor de la tensión psicológica y como agente que incrementa la agresión y los sentimientos de poder.

Al considerar las diferencias por sexo, únicamente las hubo en una subescala de expectativas, los hombres tuvieron mayores expectativas de que el consumo de alcohol incremente su conducta sexual que las mujeres $(\mathrm{F}=4.70, \mathrm{gl}=1, p<0.05)$. Asimismo, la interacción entre la variable sexo y problemas asociados fue igualmente significativa $(\mathrm{F}=5.231, \mathrm{gl}=1, p<0.05)$.

De igual manera, las puntuaciones en expectativas fueron más elevadas entre quienes señalaron problemas asociados con el consumo de alcohol en contraste con quienes no los reportaron, siendo las diferencias estadísticamente significativas en las seis subescalas referidas.

\section{Discusión}

Estudios previos sobre expectativas sugieren que los efectos que las personas atribuyen al consumo de al-

\section{Cuadro IV \\ ReLACIÓn ENTRE EXPECTATIVAS/CONSUMO/SEXo Y PROBLEMAS ASOCIADOS AL CONSUMO DE ALCOHOL. Instituto Nacional de Psiquiatría, México, 1998}

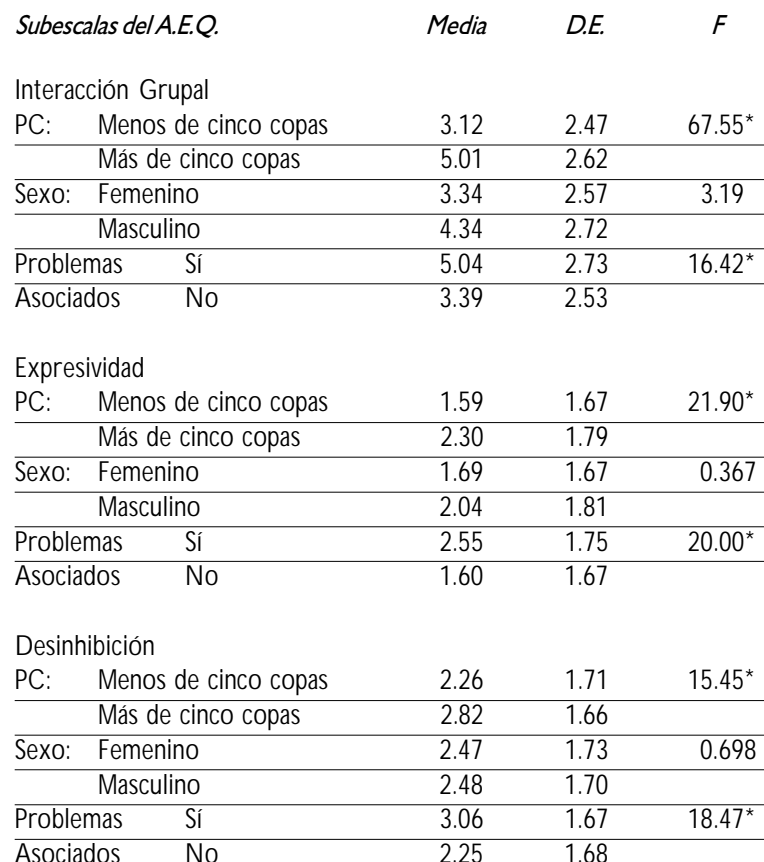

Incremento de la sexualidad"

\begin{tabular}{|c|c|c|c|c|}
\hline PC: & Menos de cinco copas & 1.40 & 1.84 & $40.13^{*}$ \\
\hline & Más de cinco copas & 2.61 & 2.40 & \\
\hline Sexo: & Femenino & 1.46 & 1.93 & $4.55^{\ddagger}$ \\
\hline & Masculino & 2.26 & 2.29 & \\
\hline Probl & nas & 2.70 & 2.43 & $12.49 *$ \\
\hline$\overline{\mathrm{AsOCi}}$ & dos $\quad$ No & 1.54 & 1.95 & \\
\hline
\end{tabular}

Tensión Psicológica

\begin{tabular}{|c|c|c|c|c|}
\hline PC: Men & inco copas & 1.66 & 1.76 & $67.71^{*}$ \\
\hline & cinco copas & 3.05 & 1.90 & \\
\hline Sexo: & & 1.85 & 1.92 & 1.41 \\
\hline & & 2.54 & 1.90 & \\
\hline Problem & Sí & 2.96 & 1.94 & $9.62^{5}$ \\
\hline A sociad & No & 1.91 & 1.86 & \\
\hline
\end{tabular}

Agresividad y poder

\begin{tabular}{|c|c|c|c|c|}
\hline PC: & Menos de cinco copas & 1.68 & 2.16 & $29.22^{*}$ \\
\hline & Más de cinco copas & 2.83 & 2.55 & \\
\hline Sexo: & Femenino & 1.83 & 2.20 & 0.95 \\
\hline & Masculino & 2.41 & 2.51 & \\
\hline Proble & mas $\quad$ Sí & 3.39 & 2.65 & $41.25^{*}$ \\
\hline Asocia & dos No & 1.63 & 2.07 & \\
\hline
\end{tabular}

$* p<0.001$,

${ }^{\ddagger} p<0.005$,

$\S p<0.05$

\# La interacción entre las variables sexo y problemas asociados fue significativa $(\mathrm{F}=5.23, \mathrm{gl}=1, p<0.05)$.

$P C$ : patrón de consumo

$n=678$ 
cohol, anticipándose a la experiencia de estar bebiendo, parecen jugar un papel central como un factor que se encuentra estrechamente relacionado con el consumo de alcohol.

De manera consistente con lo que se ha documentado en otras investigaciones, los resultados de este estudio indican que los estudiantes con un consumo alto de alcohol y los que reportaron problemas asociados al consumo tuvieron mayores puntuaciones en las subescalas de expectativas, con excepción de las áreas de reducción de la tensión física y cambios psicofisiológicos.

La principal expectativa asociada con el consumo fue el alcohol como facilitador de la interacción social, tanto en consumidores altos como en moderados, lo cual tiene que ver indudablemente con la gran tolerancia social hacia el consumo que existe en nuestra cultura. En ese sentido, el contexto social del consumo juega un papel significativo en las experiencias reforzadoras del consumo de alcohol..$^{22}$ Los hallazgos de investigación en esta línea sugieren que las expectativas sobre los efectos sociales del consumo pueden ser importantes predictores de un consumo temprano en los jóvenes. ${ }^{8}$

Los resultados del presente estudio indican de manera consistente, con lo previamente informado, que las expectativas del alcohol como reductor de la tensión y como agente que incrementa la conducta agresiva probablemente se relacionan con un consumo de alcohol más problemático, tal y como se ha encontrado en otros estudios en población alcohólica y en consumidores excesivos. ${ }^{1,3}$

Otro aspecto importante a considerar es la manera como las expectativas y la conducta de consumo son moderadas por diversas variables como el género. En el presente estudio se encontraron diferencias únicamente en las creencias relacionadas con la sexualidad, siendo los varones quienes esperan obtener un mejor desempeño sexual tanto en sí mismos como en personas del sexo opuesto a través de la ingesta de alcohol, lo cual ha sido una tendencia que se encuentra estrechamente relacionada con la edad; a medida que ésta se incrementa en los consumidores disminuyen las expectativas sexuales relacionadas con el consumo. ${ }^{23}$

Esta investigación constituye una aproximación inicial al estudio de las expectativas y cuenta con una serie de limitaciones que deberán ser consideradas en futuras investigaciones. Es necesario identificar algunas de las variables más importantes que se relacionan con el proceso de consumo, tales como a) los cambios en las expectativas a partir de las cantidades de alcohol que se emplean, b) el contexto social que refuerza el consumo; ${ }^{23} \mathrm{c}$ ) la historia familiar del uso y abuso; d) las motivaciones al consumo y razones para seguir bebiendo, ${ }^{24}$ e) el contraste en las expectativas en población bebedora y no bebedora, f) las expectativas como moderadoras de estilos de enfrentamiento, y g) la búsqueda de nuevas sensaciones y conductas de riesgo. ${ }^{25}$

Otro reto es abordar las expectativas en población infantil puesto que diferentes investigaciones han puesto de manifiesto la existencia de expectativas a edades muy tempranas, entre los 5 y 12 años. ${ }^{12}$

Leigh propone que estas creencias respecto a los efectos del alcohol sobre el comportamiento, estado de ánimo y emociones no deben ser vistas sólo como mediadoras de los efectos del alcohol, sino como factores de riesgo para la iniciación y mantenimiento del consumo. De allí la importancia de destacar la utilidad del cuestionario de expectativas como un instrumento tanto clínico como de investigación sobre alcohol. $^{5}$

Las expectativas y su importancia como variables psicosociales potencialmente modificables tienen implicaciones en el ámbito preventivo en la población de estudio. Es necesario dirigir la atención no sólo hacia los estudiantes que presentan un consumo de alto riesgo, reorientando sus cogniciones a través de información con el objeto de canalizar sus respuestas de enfrentamiento hacia otras acciones que no involucren el consumo excesivo, ${ }^{26}$ sino incorporar incluso a los jóvenes que presentan un consumo moderado, mediante un balance más adecuado entre expectativas positivas y negativas asociadas con el consumo de alcohol. ${ }^{27}$

\section{Agradecimientos}

Nuestro reconocimiento a las personas que apoyaron esta investigación, a la socióloga Rosario Villalvazo (servicio social), a las psicólogas Aline Bocardo y Sandra Ducoing, quienes participaron en el trabajo de campo y al licenciado Jorge Villatoro, por la asesoría estadística, y principalmente a los informantes por su valiosa colaboración.

\section{Referencias}

1. Brown SA, Goldman M, Christiansen BA. D o alcohol expectancies mediate drinking patterns of adults? J C onsult C lin Psychol 1985;53:512-519. 2. Goldman MS, Brown SA, Christiansen BA. Expectancy theory: Thinking about drinking. En: Blane HT, Leonard KE, ed. Psychological theories of drinking and alcoholism N ueva York: Guilford Press, 1987:181-226.

3. Brown SA, Goldman MS, Inn A, Anderson LR. Expectations of reinforcement from alcohol their domain and relation to drinking patterns. J Consult Clín Psychol 1980;48:419-426. 
4. Brown SA. Expectancies versus background in the prediction of college drinking patterns. J Consult Clin Psychol 1985;53:123-130.

5. Brown S, Christiansen BA, Goldman M. The alcohol expectancy questionnaire: An instrument for the assessment of adult alcohol expectancies. J Stud Alcohol 1987;48(5):483-490.

6. Southwick L, Steele C, Marlatt GA, Lindell M. Alcohol-related expectancies: D efined by phase of intoxication and drinking experience. J Consult Clin Psychol 1981;49:713-721.

7. Rohsenow D. Drinking habits and expectancies about alcohol's effects for self versus others. J Consult Clin Psychol 1983;51:752-756.

8. Critchlow LB. Belief about the effects of alcohol on self and others. J Stud Alcohol 1987;48(5):467-475.

9. Leigh BC. Confirmatory factor analysis of alcohol expectancy scales. Journal of Studies on Alcohol 1989;50:268-277.

10. Stacy A, W idaman K, Marlatt A. Expectancy models of alcohol use. J Pers Soc Psychol 1990;58(5):918-928.

11. Kline RB. The relation of alcohol expectancies to drinking patterns among alcoholics: Generalization across gender and race. J Stud Alcohol 1990;51(2):175-182.

12. Miller PM, Smith GT, Goldman MS. Emergence of alcohol expectancies in Childhood: A possible critical period. I Stud Alcohol 1992;51:343-349. 13. Reese $F, C$ hassin L, Molina B. Alcohol expectancies in early adolescents: Predicting drinking behavior from alcohol expectancies and parental alcoholism. J Stud Alcohol 1994;55:276-284.

14. Christiansen BA, Smith GT, Roehling P, Goldman M. Using alcohol expectancies to predict adolescent drinking behavior after one year. J Consult Clin Psychol 1989;57:93-99.

15. G arcía-Andrade C, W all TL, Ehlers L. Alcohol expectancies in a native American population. Alcohol: Clin Exp Res 1996;20(8):1438-1442.
16. Gilbert JM, Mora J, Ferguson LR. Alcohol related expectations among Mexican-american women. Int J Addict 1994;29(9):1127-1147.

17. C astro ME, Maya MA. El consumo de alcohol en la población estudiantil. Salud Mental 1987;10(4):52-58.

18. Medina-Mora ME. Los conceptos de uso, abuso, dependencia y su medición. En: Conyer T. Las adicciones, dimensión, impacto y perspectivas. México D.F.: El Manual Moderno, 1994, pp.25-55.

19. Mora J, N atera G, Villatoro J, Villalvazo R. Validez factorial del cuestionario de expectativas hacia el alcohol en estudiantes universitarios. Psicología Conductual 2000;8(2).

20. Secretaría de Salud. Encuesta N acional de Adicciones (EN A-1988). México, D.F.: Secretaría de Salud- Dirección General de Epidemiología/ Instituto Mexicano de Psiquiatría.

21. Bentler, PM. EQ S Structural Equations Program Manual, 1995. Multivariate Software, Inc. Encino.

22. Brown S. Context of drinking and reinforcement from alcohol: Alcoholic patterns. Addict Behav 1985;10(2):191-195.

23. Critchlow $L B$. The relationship of sex-related alcohol expectancies to alcohol consumption and sexual behaviour. Br J Addict 1990;85:919-928. 24. Lemmens PH, D ubois W. Reasons for drinking and alcohol expectancies in a D utch sample of middle aged men and women. Twenty fifth Annual Alcohol Epidemiology Symposium; 1999 june; Montreal Canada.

25. Cooper LM, Russell M, Skinner JB, Frone MR, Mudar P. Stress and alcohol use: Moderating effects of gender, coping and alcohol expectancies. J Abnormal Psychol 1992;101(1):139-152.

26. Henderson M, Goldman M, Coovert MD, Cannevalla N. Covariance structure models of expectancy. I Stud Alcohol 1994,55:315-326.

27. Ayala H. El curso del alcoholismo: una visión psicológica. Investigación Psicológica 1993;3:5-36. 